\section{Feasibility and Efficacy of Fusion Imaging Systems for Immediate Post Ablation Assessment of Liver Neoplasms: Protocol for a Rapid Systematic Review}

\author{
PRAGATI RAI (1)
}

SARADA DAKUA (1)

JULIEN ABINAHED (1)

SHIDIN BALAKRISHNAN D

*Author affiliations can be found in the back matter of this article

\section{ABSTRACT}

Introduction: Percutaneous thermal ablation is widely adopted as a curative treatment approach for unresectable liver neoplasms. Accurate immediate assessment of therapeutic response post-ablation is critical to achieve favourable outcomes. The conventional technique of side-by-side comparison of pre- and post-ablation scans is challenging and hence there is a need for improved methods, which will accurately evaluate the immediate post-therapeutic response.

Objectives and Significance: This review summarizes the findings of studies investigating the feasibility and efficacy of the fusion imaging systems in the immediate post-operative assessment of the therapeutic response to thermal ablation in liver neoplasms. The findings could potentially empower the clinicians with updated knowledge of the state-of-the-art in the assessment of treatment response for unresectable liver neoplasms.

Methods and Analysis: A rapid review will be performed on publicly available major electronic databases to identify articles reporting the feasibility and efficacy of the fusion imaging systems in the immediate assessment of the therapeutic response to thermal ablation in liver neoplasms. The risk of bias and quality of articles will be assessed using the Cochrane risk of bias tool 2.0 and Newcastle Ottawa tool.

Ethics and Dissemination: Being a review, we do not anticipate the need for any approval from the Institutional Review Board. The outcomes of this study will be published in a peer-reviewed journal.

\section{Highlights}

Evaluation of the therapeutic response in liver neoplasms immediately post-ablation is critical to achieve favourable patient outcomes. We will examine the feasibility and technical efficacy of different fusion imaging systems in assessing the immediate treatment response post-ablation. The findings are expected to guide the clinicians with updated knowledge on the state-of-the-art when assessing the immediate treatment response for unresectable liver neoplasms.

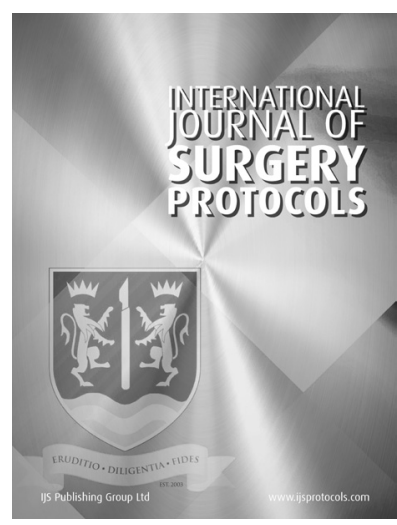

PROTOCOL

阅娄 IJS Press

Part of the IJS Publishing Group
CORRESPONDING AUTHOR:

\section{Shidin Balakrishnan}

Department of Surgery, Surgical Research Section, Hamad General Hospital, Hamad Medical Corporation, Doha, PO Box 3050, Qatar

sbalakrishnan1@hamad.qa

\section{KEYWORDS:}

Liver neoplasms; Ablation techniques; Treatment outcomes; Ablative margin; Image fusion

TO CITE THIS ARTICLE: Rai P, Dakua S, Abinahed J, Balakrishnan S. Feasibility and Efficacy of Fusion Imaging Systems for Immediate Post Ablation Assessment of Liver Neoplasms: Protocol for a Rapid Systematic Review. International Journal of Surgery: Protocols. 2021; 25(1), pp. 209-215. DOI: https://doi. org/10.29337/ijsp.162 


\section{INTRODUCTION}

Image-guided percutaneous ablation is being recognized as the curative option for unresectable liver neoplasms [1]. Patients treated with thermal ablation are usually associated with fewer postoperative complications, infection and reduced hospital stays as compared to those undergoing surgical resection [2]. Despite potential advantages, the therapeutic effect of ablation is hampered by high rates of local tumor progression (LTP) [3-6]. Factors leading to LTP include suboptimal ablative safety margin, large tumor size, tumor proximity to the blood vessel, subcapsular location and adhesion of viable tumor cells to the RFA electrodes [7].

Since intraoperative histological confirmation of the ablation zone is not possible, it becomes critical to evaluate the therapeutic outcome of the ablation immediately post-ablation to achieve the most favourable outcomes [8]. Conventionally, the therapeutic outcome of ablation is evaluated through the presence/absence of residual tumor on first follow-up or the absence of LTP in subsequent follow-up imaging [9]. Hence, an immediate intraprocedural technique to predict the treatment success would be highly desirable, due its potential clinical impact [8]. This would allow the clinicians to immediately assess the treatment response post-procedure and perform immediate supplementary ablation if needed. This potentially avoids the need for secondary treatment as well as reducing the economic burden to the patients [10].

A crucial step in the curative treatment of liver neoplasms is the evaluation of treatment success [11]. Pre-ablation planning using volumetric assessment from Computed tomography(CT)/Magnetic resonance(MR) images can positively impact treatment success [12-14]. However, immediate post-ablative assessment would provide a more accurate clinical picture on the efficacy of the treatment. Accumulating evidence suggests that ablative margin (AM) is an independent predictor of LTP and a critical determinant affecting treatment success [15-17]. There have been efforts to assess AM by manual and visual comparison of pre- and postablation two-dimensional (2D) images [18, 19]. However, this technique is subjective and prone to errors and can be challenging even for experienced radiologists. Hence, there is a clear need for methods for the precise evaluation of the AM immediately after ablation [20].

In recent years, novel imaging methods have emerged, which utilize image fusion techniques for post-ablation assessment, which can then be visualized using augmented-and mixed-reality devices [21]. These fusion techniques include CT-CT, MR-MR, ultrasound (US)-CT/MR and contrast-enhanced ultrasound (CEUS)US in the assessment of AM [22-24]. New emerging supportive software platforms are also available that reliably and quickly assess the ablation areas and the extent of the AM. In these emerging platforms, the preand post-ablation images are merged using either rigid or nonrigid registration software $[25,26]$. Image fusion using commercially available registration software also assess the AM that can be used as an intraoperative tool to evaluate the treatment efficacy of ablation procedures [27]. This work aims to summarise studies investigating the feasibility and efficacy of the fusion imaging systems for the immediate assessment of the therapeutic response to thermal ablation in liver neoplasms.

\section{OBJECTIVES AND SIGNIFICANCE}

Tumor-free margin as well as the complete eradication of microscopic invasion around the pathological periphery is critical for an optimal treatment outcome while maintaining liver function. Therefore, immediate accurate assessment of the ablative safety margin is a crucial step in clinical practice, as it may influence the following treatment or follow-up strategy. We propose a rapid review overviewing the feasibility and efficacy of the fusion imaging systems in the immediate assessment of the therapeutic response to thermal ablation in liver neoplasms. It is expected that the findings of this study could potentially empower the clinicians with updated knowledge of the state-of-the-art image fusion techniques in the assessment of treatment response for unresectable liver neoplasms immediately post ablation.

In this regard, we shall attempt to respond to the following questions:

- Are fusion imaging systems feasible for assessing the immediate therapeutic response to thermal ablation in liver neoplasms?

- Do fusion imaging systems accurately detect the AM to determine the technical efficacy of thermal ablation in liver neoplasms?

\section{METHODS AND ANALYSIS 3.1 STUDY DESIGN}

This review will be designed and implemented based on the Preferred Reporting Items for Systematic Reviews and Meta-Analyses (PRISMA) guidelines [28]. The research question is formulated within the PICO tool, as follows:

(P) Participants: Liver neoplasms (primary or secondary) treated with Thermal ablation [Radiofrequency ablation (RFA)/Microwave ablation (MWA)].

- (I) Intervention: Assessment of therapeutic response in liver neoplasms immediately post ablation by fusion of pre-(CT or MR or US) ablation and post (CT or MRI or CEUS) ablation images. Qualitative and quantitative assessment of AM done immediately at the end of ablation by fusion imaging systems. 
- (C) Comparator: None.

- (O) Outcome: Feasibility and technical efficacy of fusion imaging systems for assessment of AM immediately after percutaneous thermal ablation of liver neoplasms.

This rapid review protocol has been registered in the International Prospective Register for Systematic Reviews and Meta-analysis (PROSPERO) with the registration number CRD42021265980.

\subsection{ELIGIBILITY CRITERIA}

For this review, we will consider studies following experimental as well as observational study design, specifically controlled trials (randomized and nonrandomized), cohort studies (prospective and retrospective), and analytical case-control and crosssectional studies. Based on preliminary analysis, the authors determined the time for the search to be from January 2016 to June 2021. From these, those studies will be included which specifically assess the immediate therapeutic response to percutaneous thermal ablation in liver neoplasms using fusion imaging systems. Any descriptive or non-analytical observational studies/commentaries will be discarded, including case reports/series, review articles, letters, consensus statements and opinions. Additionally, liver neoplasms treated with techniques other than RFA and MWA will be excluded. Articles published in languages other than English will be not be included.

\subsection{SEARCH STRATEGY}

The electronic database MEDLINE (via PUBMED), EMBASE and Cochrane Library Central Registry databases will be extensively searched to retrieve articles related to the application of fusion imaging in the evaluation of immediate therapeutic response to ablation for liver neoplasms. Full-text papers will be obtained after the abstracts are evaluated for relevance. The reference lists of included articles will be manually searched to identify any additional studies. To maximize the sensitivity of the search, we will use both Medical Subject Headings (MeSH) and keyword searches. The search terms "liver neoplasms" [MeSH Terms], "carcinoma, hepatocellular" [MeSH Terms], "ablation techniques" [MeSH Terms], "treatment outcome” [MeSH Terms] 'ablative margin', 'fusion imaging', 'intraprocedural', 'three dimensional' and 'volumetric assessment' will be used in combination with the Boolean operators AND or OR.

\subsection{STUDY SELECTION}

After applying the search criteria above, selected articles will be exported to Covidence software and any duplicates will be removed [29]. Three reviewers (PR, SB, SD) will independently screen title and abstract of the studies based on the predefined inclusion and exclusion criteria. Regular meetings will be held to discuss potential conflicts, and any disagreements will be resolved through discussion with a fourth reviewer (JA).

Following that, full text of selected papers will be screened for further selection. Reasons for exclusion of full-text papers that do not fulfil the inclusion criteria will be recorded and reported in the review. The final selected list of articles will concurrently be evaluated by the fourth reviewer (JA) against the predefined inclusion criteria, and any conflicts would be resolved through discussions. The results of the search will be reported in the final review and presented as a PRISMA flow diagram.

\subsection{DATA EXTRACTION}

Data from selected articles will be assessed and analyzed independently by three reviewers (PR, SB, SD) using the data extraction capabilities of the Covidence platform. Data extraction will be based on a set of predefined variables/labels. From each selected study, baseline patient characteristics (age, tumor type, tumor size), study design, ablation type, type of fusion modality employed, technical efficacy, time taken for fusion of preand post-ablation images, software used for assessment of $\mathrm{AM}$ ), complete ablation rate, supplementary ablation rate, follow up time, imaging modality used during followup and LTP rates will be recorded. Any modifications to the extraction protocol will be discussed with the fourth reviewer (JA).

\subsection{ASSESSMENT OF RISK OF BIAS}

The observational studies will be critically evaluated for methodological quality using the Newcastle Ottawa scale [30]. The Cochrane risk of bias 2.0 tool will be used to assess the risk of bias for interventional studies [31]. If required, the authors of the papers would be contacted for any additional clarifications or data. The results of critical appraisal will be reported in risk of bias summary and table. Any disagreements or discordance amongst the authors will be resolved through discussion with the fourth author (JA).

\subsection{DATA PRESENTATION AND DATA SYNTHESIS}

The extracted data will be presented in diagrammatic and tabular format in a manner that aligns with the objective of this review. A narrative synthesis of the findings will be provided from the included studies, structured around the feasibility and technical efficacy of different fusion imaging systems for the immediate assessment of AM post-ablation in liver neoplasms. Differences in outcomes based on patient characteristics (age, tumor type, tumor size) would be highlighted. Comparative synthesis of ablation type and type of fusion modality employed would be undertaken, and their impact on technical efficacy and time taken would be analyzed. Any influence of software used for assessment of AM on complete ablation rate, supplementary ablation rate would be analyzed. Duration of follow-up, imaging modality used during follow-up and LTP rates will be explored. 


\section{ETHICS AND DISSEMINATION}

This review uses peer reviewed published literature and attempts to summarize their findings narratively; as a result, we do not anticipate any need for an institutional review board approval. The results of this study will be summarised in English and will be published in a peer-reviewed scientific publication and disseminated on research platforms in accordance with copyright regulations.

\section{DISCUSSION}

\subsection{CURRENT IMPLICATIONS OF THE STUDY}

Tissue changes at the ablative site evolves with time after the procedure. This makes it challenging to accurately assess the post-ablative efficacy of treatment as more time passes after the procedure. Tumor-free margin and total eradication of microscopic invasion around the pathological periphery is critical for optimal treatment outcome while maintaining hepatic function. Therefore, immediate accurate assessment of the ablative safety margin is crucial in clinical practice, as it may impact the subsequent treatment or follow-up strategy

The findings of the current review will potentially empower the clinicians with updated knowledge of the state-of-the-art image fusion techniques in the assessment of treatment response for unresectable liver neoplasms immediately post-ablation. Application of fusion of pre- and post-interventional scans in conjunction with an assessment of the AM immediately post-ablation can possibly prevent any errors and objectively evaluate the technical success of the procedure. This approach could have a potential clinical impact in identifying possible sub-total ablation and the need for re-treatment within the same operative session. By reducing additional treatment sessions, it could improve patient's quality of life, decrease stress, and ease their financial burden as well as optimize usage of hospital resources.

\subsection{LIMITATIONS OF THE STUDY}

The study is anticipated to have some limitations in terms of concurrent clinical practice and research. There is limited analytic literature reported on the immediate post-operative assessment of AM after ablation using fusion imaging techniques, which would directly affect our synthesis. The different fusion imaging techniques used to assess immediate post-ablative margins have yet to reach consensus and standardization among clinicians. Only a few comparative studies between the different fusion imaging techniques have been done to assess immediate post therapeutic response. There is also a lack of consensus on a standardized protocol between clinicians for the same registration software used for the quantitative assessment of AM. The current protocol did not incorporate grey literature, where the most current and updated emerging techniques are first published. Further research is also required to validate the use of existing commercial co-registration software (Ablation-fit TM [32], Hepacare [33] etc.) to assess AM intra-procedurally for a broader group of tumour types (subcapsular tumors, subphrenic tumors, etc.) and ablation procedures (cryoablation, laser ablation, etc).

Finally, despite technological advancements, fusion registration still faces technical challenges and limitations with the existing methods in the setting of organ motion induced by breathing or positional changes [34-39]. These challenges include (a) different physical acquisition processes, which may generate a statistical correlation between imaging structures that do not correspond to the same anatomical structures, violating one of the underlying assumptions for most intensity-based similarity measures, and (b) the deformation, spatial and temporal variabilities.

\subsection{FUTURE IMPLICATIONS OF THE STUDY}

The study could potentially highlight areas of future research addressing primarily the technical limitations due to deformation, spatial and temporal variabilities, to gain widespread clinical acceptance of robust techniques. The ability to capture complex image deformations and establish accurate pointwise correspondence is key to many clinical applications of computer vision that involve image fusion and atlas construction. These properties become particularly challenging when the object depicted on the images undergoes a severe deformation or has in general a high shape variability. Addressing dissimilarities due to inter- and intra-fractional anatomical variations from the pre-operative image set can further augment accuracy of the technical fusion pipeline, empowering widespread use in clinical practice. Researching techniques to homogenize multi-modality, multi-temporality, domain specificity and parameter sensitivity may facilitate image processing algorithms for segmentation, rigid and non-rigid co-registration, and volume analysis devoted to intraprocedural quantitative assessment of AM. This study could also highlight research focus for the refinement of breathing synchronization devices and automatic recognition and registration of hepatic vessels.

\section{AMENDMENTS}

Any amendments to this protocol will be prospectively updated on the PROSPERO International Prospective Register of Systematic Reviews. 


\section{ABBREVIATIONS}

PRISMA-P: Preferred Reporting Items for Systematic Review and Meta-Analysis Protocols

AM: Ablative margin

LTP: Local tumor progression

CT: Computed tomography

MR: Magnetic resonance

US: Ultrasound

CEUS: Contrast-enhanced ultrasound

\section{ETHICS AND CONSENT}

As this is a review, we do not anticipate the need for any approval from the Institutional Review Board as no patient data/identifiers will be disclosed.

\section{ACKNOWLEDGEMENTS}

This publication was made possible by NPRP11S-1219-170106 from the Qatar National Research Fund (a member of Qatar Foundation). The findings herein reflect the work, and are solely the responsibility of the authors.

\section{COMPETING INTERESTS}

The authors have no competing interests to declare.

\section{AUTHOR CONTRIBUTIONS}

PR, SD and SB conceptualised the study. PR and SB drafted the initial manuscript. JA and SD made critical revisions. All authors approved the final submission.

\section{AUTHOR AFFILIATIONS}

\section{Pragati Rai (D) orcid.org/0000-0003-2569-9332}

Department of Surgery, Hamad Medical Corporation, Doha, Qatar

Sarada Dakua (D) orcid.org/0000-0003-3193-4343

Department of Surgery, Hamad Medical Corporation, Doha, Qatar

Julien Abinahed (D) orcid.org/0000-0002-6386-4813

Department of Surgery, Hamad Medical Corporation, Doha, Qatar

Shidin Balakrishnan (D) orcid.org/0000-0001-6361-4980

Department of Surgery, Hamad Medical Corporation, Doha, Qatar

\section{REFERENCES}

1. Yoon JH, Lee JM, Klotz E, Woo H, Yu MH, Joo I, Lee ES, Han JK. "Prediction of local tumor progression after radiofrequency ablation (RFA) of hepatocellular carcinoma by assessment of ablative margin using Pre-RFA MRI and post-RFA CT registration." Korean Journal of Radiology. 2018; 19(6): 1053-1065. DOI: https://doi.org/10.3348/ kjr.2018.19.6.1053

2. Gong Y, Tang Y, Geng Y, Zhou Y, Yu M, Huang B, Sun Z, Tang H, Jian Z, Hou B. "Comparative safety and effectiveness of ultrasound-guided radiofrequency ablation combined with preoperative three-dimensional reconstruction versus surgical resection for solitary hepatocellular carcinoma of 3-5 cm." Journal of Cancer. 2019; 10(22): 5568-5574. DOI: https://doi.org/10.7150/jca.32342

3. Zhihua L, Yukun Z, Peng Z, Huanlong Q. "Metaanalysis of the therapeutic effect of hepatectomy versus radiofrequency ablation for the treatment of hepatocellular carcinoma." Surgical laparoscopy, endoscopy \& percutaneous techniques. 2010; 20(3): 130-140. DOI: https://doi.org/10.1097/SLE.0b013e3181d823df

4. Jiwei $\mathbf{H}$, Lvnan $Y$, Zheyu C, Hong $\mathbf{W}$, Liang D, Jinzhou W, Yinglong $\mathbf{X}$, Yong Z. "A randomized trial comparing radiofrequency ablation and surgical resection for HCC conforming to the Milan criteria." Annals of surgery. 2010; 252(6): 903-912, DOI: https://doi.org/10.1097/ SLA.0b013e3181efc656

5. Li K, Su ZZ, Xu EJ, Ju JX, Meng XC, Zheng RQ.

"Improvement of ablative margins by the intraoperative use of CEUS-CT/MR image fusion in hepatocellular carcinoma." BMC Cancer. 2016; 16(1): 1-9. DOI: https://doi.org/10.1186/ s12885-016-2306-1

6. Yi Hm, Zhang W, Ai X, Li KY, Deng YB. "Radiofrequency ablation versus surgical resection for the treatment of hepatocellular carcinoma conforming to the Milan criteria: systemic review and meta-analysis." International journal of clinical and experimental medicine. 2014. [Online]. 7(10): 315063. Available: https://pubmed.ncbi.nlm.nih.gov/25419346/.

7. Hendriks P, Noortman WA, Baetens TR, Van Erkel AR, Van Rijswijk CSPR, Van Der Meer W, Coenraad MJ, De Geus-Oei LF, Slump CH, Burgmans MC. "Quantitative Volumetric Assessment of Ablative Margins in Hepatocellular Carcinoma: Predicting Local Tumor Progression Using Nonrigid Registration Software." Journal of Oncology; 2019. DOI: https://doi.org/10.1155/2019/4049287

8. Laimer G, Schullian P, Jaschke N, Putzer D, Eberle G, Alzaga A, Odisio B, Bale R. "Minimal ablative margin (MAM) assessment with image fusion: an independent predictor for local tumor progression in hepatocellular carcinoma after stereotactic radiofrequency ablation." European Radiology. 2020; 30(5): 2463-2472. DOI: https://doi. org/10.1007/s00330-019-06609-7

9. Hocquelet A, Trillaud H, Frulio N, Papadopoulos $P$, Balageas P, Salut C, Meyer M, Blanc JF, Montaudon M, Denis De Senneville B. "Three-Dimensional Measurement of Hepatocellular Carcinoma Ablation Zones and Margins for Predicting Local Tumor Progression.” Journal of Vascular and Interventional Radiology. 2016; 27(7): 1038-1045.e2. DOI: https://doi.org/10.1016/j.jvir.2016.02.031 
10. Ma QP, Xu EJ, Zeng QJ, Su ZZ, Tan L, Chen JX, Zheng RQ, Li K. "Intraprocedural computed tomography/magnetic resonance-contrast-enhanced ultrasound fusion imaging improved thermal ablation effect of hepatocellular carcinoma: Comparison with conventional ultrasound." Hepatology Research. 2019; 49(7): 799-809. DOI: https:// doi.org/10.1111/hepr.13336

11. Laimer G, Schullian P, Putzer D, Eberle G, Goldberg SN, Bale R. "Can accurate evaluation of the treatment success after radiofrequency ablation of liver tumors be achieved by visual inspection alone? Results of a blinded assessment with 38 interventional oncologists." International Journal of Hyperthermia. 2020; 37(1): 1362-1367. DOI: https://doi.org/ 10.1080/02656736.2020.1857445

12. Dakua SP, Abinahed J, Al-Ansari AA. "Pathological liver segmentation using stochastic resonance and cellular automata." Journal of Visual Communication and Image Representation. 01/01/2016; 34: 89-102. DOI: https://doi. org/10.1016/j.jvcir.2015.10.016

13. Dakua SP, Abinahed J, Al-Ansari A. "Semiautomated hybrid algorithm for estimation of three-dimensional liver surface in CT using dynamic cellular automata and levelsets." (in eng). J Med Imaging (Bellingham). Apr 2015; 2(2): 024006. DOI: https://doi.org/10.1117/1.JMI.2.2.024006

14. Akhtar Y, Dakua SP, Abdalla A, Aboumarzouk OM, Ansari MY, Abinahed J, Elakkad MSM, Al-Ansari A. "Risk Assessment of Computer-aided Diagnostic Software for Hepatic Resection." IEEE Transactions on Radiation and Plasma Medical Sciences. 2021; 1-1. DOI: https://doi. org/10.1109/TRPMS.2021.3071148

15. Zytoon AA, Ishii H, Murakami K, El-Kholy MR, Furuse J, El-Dorry A, El-Malah A. "Recurrence-free survival after radiofrequency ablation of hepatocellular carcinoma. $A$ registry report of the impact of risk factors on outcome." Japanese journal of clinical oncology. 2007; 37(9): 658-672. DOI: https://doi.org/10.1093/jjco/hym086

16. Nakazawa T, Kokubu S, Shibuya A, Ono K, Watanabe M, Hidaka H, Tsuchihashi T, Saigenji K. "Radiofrequency ablation of hepatocellular carcinoma: correlation between local tumor progression after ablation and ablative margin." AJR. American journal of roentgenology. 2007; 188(2): 480-488, DOI: https://doi.org/10.2214/AJR.05.2079

17. Li K, Su Z, Xu E, Huang Q, Zeng Q, Zheng R. "Evaluation of the ablation margin of hepatocellular carcinoma using CEUS-CT/MR image fusion in a phantom model and in patients." BMC Cancer. 2017; 17(1): 1-10. DOI: https://doi. org/10.1186/s12885-017-3061-7

18. Jeon SH, Cho YK, Choi SA, Kim MY, Lee HS. "Analysis of Factors Affecting Local Tumor Progression of Colorectal Cancer Liver Metastasis after Radiofrequency Ablation." Journal of the Korean Society of Radiology. 2017; 76(3): 179-186. DOI: https://doi.org/10.3348/ jksr.2017.76.3.179

19. Shady W, Petre EN, Do KG, Kingham PT, Solomon SB, Sofocleous CT. "Percutaneous Microwave versus Radiofrequency Ablation of Colorectal Liver Metastases:
Ablation with Clear Margins (A0) Provides the Best Local Tumor Control." Journal of vascular and interventional radiology: JVIR. 2018; 29(2): 268-275.e1. DOI: https://doi. org/10.1016/j.jvir.2017.08.021

20. Kaye EA, Cornelis FH, Petre EN, Tyagi N, Shady W, Shi W, Zhang Z, Solomon SB, Sofocleous CT, Durack JC. "Volumetric 3D assessment of ablation zones after thermal ablation of colorectal liver metastases to improve prediction of local tumor progression." European Radiology. 2019; 29(5): 2698-2705. DOI: https://doi.org/10.1007/ s00330-018-5809-0

21. Halabi O, Balakrishnan S, Dakua SP, Navab N, Warfa M. "Virtual and Augmented Reality in Surgery." In Doorsamy W. Paul BS, Marwala T (eds), The Disruptive Fourth Industrial Revolution: Technology, Society and Beyond. 2020; $257-$ 285. Cham: Springer International Publishing. DOI: https:// doi.org/10.1007/978-3-030-48230-5_11

22. Sakakibara M, Ohkawa K, Katayama K, Imanaka K, Ishihara A, Hasegawa N, Kimura H. "Three-dimensional registration of images obtained before and after radiofrequency ablation of hepatocellular carcinoma to assess treatment adequacy." American Journal of Roentgenology. 2014; 202(5): 487-495. DOI: https://doi. org/10.2214/AJR.13.11384

23. Zhong-Zhen S, Kai L, Rong-Qin Z, Er-Jiao X, Ting Z, Ao-Hua Z, Shu-Fang Y, Xu-Qi H. "A feasibility study for determining ablative margin with 3D-CEUS-CT/MR image fusion after radiofrequency ablation of hepatocellular carcinoma." Ultraschall in der Medizin. 2012; 33(7). DOI: https://doi.org/10.1055/s-0032-1325466

24. Kim YS, Lee WJ, Rhim HH, Lim K, Choi D, Lee JY. "The minimal ablative margin of radiofrequency ablation of hepatocellular carcinoma ( $>2$ and $<5 \mathrm{~cm}$ ) needed to prevent local tumor progression: 3D quantitative assessment using CT image fusion." American Journal of Roentgenology. 2010; 195(3): 758-765. DOI: https://doi. org/10.2214/AJR.09.2954

25. Mauri G, Solbiati L, Orsi F, Monfardini L. "Thermal Ablation of Liver Tumours: The Crucial Role of 3D Imaging." CardioVascular and Interventional Radiology. 2020; 43(9): 1416-1417. DOI: https://doi.org/10.1007/s00270-020-02560-z

26. Sibinga Mulder BG, Hendriks P, Baetens TR, Van Erkel AR, Van Rijswijk CSP, Van Der Meer RWC, Van De Velde JH, Vahrmeijer AL, Mieog JSD, Burgmans MC. "Quantitative margin assessment of radiofrequency ablation of a solitary colorectal hepatic metastasis using MIRADA RTx on CT scans: A feasibility study." BMC Medical Imaging. 2019; 19(1): 1-8. DOI: https://doi.org/10.1186/s12880-019-0360-2

27. Solbiat M, Passera KM, Goldberg SN, Rotilio A, Ierace T, Pedicini V, Poretti D, Solbiati L. "A Novel CT to ConeBeam CT Registration Method Enables Immediate Real-Time Intraprocedural Three-Dimensional Assessment of Ablative Treatments of Liver Malignancies." Cardiovascular and interventional radiology. 2018; 41(7): 1049-1057. DOI: https://doi.org/10.1007/s00270-0181909-0 
28. Liberati A, Altman DG, Tetzlaff J, Mulrow C, Gøtzsche PC, Ioannidis JPA, Clarke M, Devereaux PJ, Kleijnen J, Moher D. "The PRISMA statement for reporting systematic reviews and meta-analyses of studies that evaluate health care interventions: explanation and elaboration." PLoS medicine. 2009; 6(7). DOI: https://doi.org/10.1371/journal. pmed.1000100

29. “Covidence." Cochrane Community. https://community. cochrane.org/help/tools-and-software/covidence (accessed).

30. Wells G, Shea B, O'Connell D, Peterson J, Welch V, Losos M, et al. The Newcastle-Ottawa Scale (NOS) for assessing the quality of nonrandomised studies in meta-analyses. http://www.ohri.ca/programs/clinical_epidemiology/oxford. asp (Accessed 16 Jan 2020). - Google Search, ed.

31. Sterne JA, Savović J, Page MJ, Elbers RG, Blencowe NS, Boutron I, Cates $\mathrm{CJ}$, Cheng HY, Corbett MS, Eldridge SM, Emberson JR, Hernán MA, Hopewell S, Hróbjartsson A, Junqueira DR, Jüni P, Kirkham JJ, Lasserson T, Li T, McAleenan A, Reeves BC, Shepperd S, Shrier I, Stewart LA, Tilling K, White IR, Whiting PF, Higgins JPT. "RoB 2: a revised tool for assessing risk of bias in randomised trials." BMJ (Clinical research ed.). 2019; 366. DOI: https://doi. org/10.1136/bmj.14898

32. Solbiati M, Muglia R, Goldberg SN, Ierace T, Rotilio A, Passera KM, Marre I, Solbiati L. "A novel software platform for volumetric assessment of ablation completeness." International Journal of Hyperthermia. 2019; 36(1): 337-343. DOI: https://doi.org/10.1080/026567 36.2019 .1569267

33. Park J, Lee JM, Lee DH, Joo I, Yoon JH, Park JY, Klotz E. "Value of Nonrigid Registration of Pre-Procedure MR with Post-Procedure CT After Radiofrequency Ablation for Hepatocellular Carcinoma." CardioVascular and
Interventional Radiology. 2017; 40(6): 873-883. DOI:

https://doi.org/10.1007/s00270-017-1571-y

34. Viergever MA, Maintz JBA, Klein S, Murphy K, Staring M, Pluim JPW. "A survey of medical image registration under review." Medical Image Analysis. 2016; 33: 140-144. DOI: https://doi.org/10.1016/J.MEDIA.2016.06.030

35. Lotz J, Olesch J, Muller B, Polzin T, Galuschka P, Lotz JM, Heldmann S, Laue H, Gonzalez-Vallinas M, Warth A, Lahrmann B, Grabe N, Sedlaczek O, Breuhahn K, Modersitzki J. "Patch-Based Nonlinear Image Registration for Gigapixel Whole Slide Images.” IEEE Transactions on Biomedical Engineering. 2016; 63(9): 1812-1819. DOI: https://doi.org/10.1109/TBME.2015.2503122

36. Li Y, Chen C, Yang F, Huang J. "Hierarchical Sparse Representation for Robust Image Registration." IEEE Transactions on Pattern Analysis and Machine Intelligence. 2018; 40(9): 2151-2164. DOI: https://doi.org/10.1109/ TPAMI.2017.2748125

37. Kéchichian R, Valette S, Desvignes M. "Automatic Multiorgan Segmentation via Multiscale Registration and Graph Cut." IEEE Transactions on Medical Imaging, Institute of Electrical and Electronics Engineers. 2018; 37(12): 1-1. DOI: https://doi.org/10.1109/TMI.2018.2851780i

38. Hu Y, Gibson E, Ahmed HU, Moore CM, Emberton M, Barratt DC. "Population based prediction of subjectspecific prostate deformation for MR-to-ultrasound image registration." Medical Image Analysis. 2015; 26(1): 332-332. DOI: https://doi.org/10.1016/J.MEDIA.2015.10.006

39. Collins JA, Weis JA, Heiselman JS, Clements LW, Simpson AL, Jarnagin WR, Miga MI. "Improving Registration Robustness for Image-Guided Liver Surgery in a Novel Humanto-hantom Data Framework." IEEE Transactions on Medical Imaging. 2017; 36(7): 1502-1510. DOI: https://doi. org/10.1109/TMI.2017.2668842

\section{TO CITE THIS ARTICLE:}

Rai P, Dakua S, Abinahed J, Balakrishnan S. Feasibility and Efficacy of Fusion Imaging Systems for Immediate Post Ablation Assessment of Liver Neoplasms: Protocol for a Rapid Systematic Review. International Journal of Surgery: Protocols. 2021; 25(1), pp. 209-215. DOI: https://doi.org/10.29337/ijsp.162

Submitted: 08 August 2021 Accepted: 02 September 2021 Published: 17 September 2021

\section{COPYRIGHT:}

(C) 2021 The Author(s). This is an open-access article distributed under the terms of the Creative Commons Attribution 4.0 International License (CC-BY 4.0), which permits unrestricted use, distribution, and reproduction in any medium, provided the original author and source are credited. See http://creativecommons.org/licenses/by/4.0/.

International Journal of Surgery: Protocols is a peer-reviewed open access journal published by IJS Publishing Group.

\section{IJS Press}

